\title{
Teaching Research on Task-Driven Method in the Application Undergraduate Human Resource Management Course
}

\author{
Lei Zhou ${ }^{1, a}$ Xiuping Liu ${ }^{1, b}$ Yuan Qian ${ }^{1, c}$ \\ ${ }^{1}$ Nanchang Institute of Science \& Technology, Nanchang, Jiangxi, China, 330000 \\ aemail, bemail, ${ }^{\mathrm{c} e m a i l,}$
}

\begin{abstract}
Keywords: Teaching Research, Task-Driven Method, Application Undergraduate, Human Resource Management Course
\end{abstract}

\begin{abstract}
Human resources management is a comprehensive management science, economics, psychology, sociology, law, talent and other fields of modern emerging management disciplines, requiring students to strengthen the practical ability and ability to solve practical problems. Combined with the characteristics of disciplines and students, you can use the task-driven theory in the human resources management curriculum for teaching reform.
\end{abstract}

\section{Introduction}

As a practical teaching method, the "task-driven method" is often used in the teaching of computer and information technology. Based on the teaching characteristics of human resource management curriculum, the author tries to apply the "task-driven method" to the teaching system of human resources management curriculum to meet the practical teaching needs of human resource management curriculum.

\section{The Connotation of "Task-Driven"}

"Task-driven" is the student with the help of teachers, closely around a common mission activity center, driven by a strong problem motive, through the active application of learning resources, self-exploration and interactive collaborative learning, and In the completion of the task at the same time, to guide students to produce a learning practice. "Task-driven approach" is a teaching method based on the theory of constructivism. It requires the "mission" of the objectives and the creation of teaching context, so that students with real tasks in the exploration of learning. "Task-driven" teaching method conforms to the inquiry teaching mode, which is suitable for cultivating students' innovation ability and independent analysis problem and problem solving ability. Human resource management curriculum features and teaching requirements Human resource management is a very practical application of the subject, this course is mainly combined with the needs of enterprises to use talent, set up human resources planning, job analysis, recruitment and selection, assessment and Incentives, pay and benefits and other aspects of the specific content. Whether it is from the labor market itself, the relationship between supply and demand caused by the discussion of the quality of modern enterprise staff, or based on the internal recruitment, training, assessment, pay system design and other aspects of the quantitative, operational requirements, this discipline in the teaching Are faced with an unprecedented challenge to the ability of students to apply. The use of "task-driven" teaching method in the teaching of human resource management is to enable students to start teaching activities in a typical task of "task", to guide students from simple to complex, from easy to difficult, step by step to complete a Series of "tasks" to get a clear idea, method and knowledge of the context, in the completion of the "task" in the process of training to analyze problems, solve problems and innovation and other aspects of the ability. At present, the "task-driven" teaching method has formed the "three main" features of "task as the main line, teacher-led, student as the main body". 


\section{The Teaching Characteristics of Human Resources Management Curriculum}

Human resources management course is a theory rich, memory knowledge more courses. Human resources management is the discipline of economics, management, psychology, sociology, philosophy and so on. It has the basic theories and methods of human resource management. The basic knowledge of higher vocational students is relatively weak. In terms of, will learn the purpose and application is not clear, have a certain weariness psychology.

At the same time, human resource management courses are also practical and highly disciplined disciplines. The practicality of human resources management courses is strong and the method is more able to combine the actual situation of enterprises and improve the ability of students to analyze and solve problems. The course requires a variety of forms and programming, as well as a variety of full-time jobs, or strong or weak, after graduation. Therefore, students also have the motivation to learn this course.

According to the concept of vocational education and teaching, the human resources management curriculum should be integrated in the vocational colleges according to the principle of "theory enough to meet the needs, strengthen the practice and focus on the application". Therefore, the curriculum can be designed by reducing the theory practice the principles of reform. The task-driven teaching method as a constructivist teaching theory can be achieved by creating a certain teaching situation and allowing students to explore through the task. Human resources management courses can be based on a number of knowledge modules, set the size of the different tasks to complete the task, through the practice of practice, both in line with the characteristics of vocational students, but also to promote their active to complete, to achieve a multiplier effect.

\section{The Practice Steps of "Task-Driven" in the Practice of Human Resources Management}

The Design of the Task. In the "task-driven", "task" is the key, so the task design is the primary task of teachers in lesson preparation. The form of the task can be derived from the examples of living in the human resources management of enterprises and institutions, but also the fictional case prepared by the teachers according to the requirements of the teaching content. In the development of scientific and reasonable learning tasks, the design of the task and the task of the task of the task should be both systematic and necessary, and follow the principle of "low threshold and slow height". Driving the fun and creativity of the task In order to receive the desired teaching effect, the proposed task must be attractive to the students. For example: in the students to teach "interview procedures and skills," the content, you can combine the professional students and the company's actual design, "TCL International Electric Chongqing branch recruitment to the most appropriate human resources director," the task, teachers you can give some advice on the basic conditions of TCL recruitment of human resources in advance, and requires students through the network, newspapers and other media search TCL International Electric's corporate background, corporate culture and so on. In this way, both to cultivate students' interest in learning, but also to play the student's initiative, so that students focus on the completion of the task up.

The Distribution of Tasks. After the task is designed, assign the task to the students. Since the number of students in a class is more reasonable, it is reasonable to allocate the tasks of the students according to the group. You can also assign the same purpose to each group, but the background of the case is different. Students are able to take on the task in the group task. Teachers assign tasks as simple as possible, concrete and easy to understand. For example, after designing TCL International's job, assign a class of students into a number of groups, each of which plays a role in the interview, such as the main test, candidate A, candidate B, candidate C, Applicant D and so on. In the main test group, but also allows the team members to play the written test designers, written examination, interview designers, interview moderators, interview participants and other roles; in the application group, the same can be based on the candidate learning experience, The background of the work so that the team members were playing different roles.

The Implementation of the Task. In this process, teachers can also choose some representative 
typical tasks, exercise from the collection of information, analysis tasks, organizational discussions, summed up to the final refinement of the whole process, so that students know how to do it. In addition, in this process, teachers should actively encourage students to adopt self-learning or collaborative learning strategies, you can also at any time through the multimedia teaching platform for electronic gestures function questions, teachers in the teacher machine to monitor student tasks to complete the progress and timely answers to questions. Specifically, combined with the above TCL International case can be from the following aspects:

Create a good learning situation. To create a good learning situation, so that students understand the necessity of learning tasks and learning tasks related to learning information, stimulate their interest in learning, so that they have the motivation to complete the task. Such as the use of multimedia in the image, animation, video and voice, text and language, symbol function, to design a "context" of learning "task" to enable students in this "context" to explore the practice, so as to deepen the problem Understand. Such as in the interview before the case, teachers use multimedia display TCL International Electric and some of the work of human resources, the screen to attract students' attention, and then put forward the task of learning, and then according to the specific objectives of the task to analyze the scene in the picture. Through such clever design, students driven by curiosity, proactively into the learning state, to complete the task of learning laid the foundation.

In the account of the task, to give students enough time to complete the task independently, "teach" division has become a veritable "guide" division, its role is to provide students with only some relevant clues to help students develop good at Brain, diligent study habits; Secondly, the use of group collaboration, the implementation of "group mutual aid" learning mechanism. After completing the learning task, the group as a unit to carry out the task of inquiry, put forward different methods of operation, which virtually improve the efficiency of the task of inquiry. It is important to note that in this session, teachers should actively participate in the identity of the flow of members of the group to understand and grasp the student's learning situation. Students in this cooperative learning to help each other, and common progress, both learned the knowledge, but also exercise the ability to collaborate, teachers also participate in group discussions and became a student of intimate friends, can be described as multiple purposes.

The Comments of the Task. The fundamental purpose of the task-driven approach is not the completion of the task assigned to each group, but rather through the completion of this task to achieve the purpose of teacher teaching, so that students master the knowledge of human resources management courses and skilled use of knowledge. The methods used in the course of the mission and the knowledge points involved, so that other groups of students understand the group to perform the task of the process used in the new method or differences, can achieve knowledge sharing, deepen understanding and learn from each other.

After the task is completed, the students' learning outcomes must be analyzed and evaluated. The best practice is to first self-evaluation within the group, and then the group between the assessment and the final teacher to add evaluation. In the process of presentation of the group, the other team members are allowed to supplement or correct, and construct a constructive and controversial view, which is conducive to students' ability to exercise reasoning, judgment, analysis and expression in communication and evaluation. In the case of TCL International Electric, the students can be included in the evaluation of the degree of participation and effects in data search, problem discussion, interview design and so on, so as to improve their enthusiasm so that they can learn from each other and learn from each other.

\section{The Problems of the "Task-Driven" Teaching in the Use of Human Resources Management}

In order to make the task-driven method to work better, in the use of task-driven method of teaching should pay attention to the following questions:

First, in the task design stage, teachers fully consider the students' existing cultural knowledge, cognitive ability, age, interest and other characteristics, follow the shallow from the deep, from the table and the gradual and other principles, pay attention to the appropriate conversion of the 
contents of the textbook or exchange, that is, "blend" task. At the same time, for new content or some difficult "task", teachers should demonstrate in advance or give a clear, detailed step to facilitate students to learn independently.

Under the guidance of the theory of constructivism, the "task-driven" teaching method requires teachers and students to change the traditional concept and role. Teachers in the teaching to play a role in organizing, guiding, promoting, controlling, consulting, which requires teachers to have a wide range of professional knowledge and profound theoretical foundation, to be good at analyzing students in the task of the implementation of the various problems and Give the right guide. Emphasizing the student's dominant position, it is necessary to give full play to students in the learning process of initiative, enthusiasm and creativity. In the teaching of human resources management, students in the organization of teachers, under the guidance of different ways to complete the activities of the "task", so that students of knowledge, thinking, skills and emotions get exercise and influence.

In order to enhance the objectivity and impartiality of the evaluation of the accomplishment of the students, it is possible for the students, teachers or professionals to participate in the evaluation by changing the practice of evaluating the students by the teachers. We should pay attention to and cultivate students' self-evaluation, encourage students to analyze and explain their own tasks and encourage them to pay attention to the feedback of other evaluation subjects. Teachers should judge the students 'learning habits, methods, emotions and attitudes, basic knowledge, practical ability and innovation consciousness by making value judgments about the accomplishment of students' tasks.

\section{Conclusion}

The practice proves that the task-driven method can promote the realization of human resource management teaching goal, and has great application value in the teaching of human resource management. Through the design, distribution, execution, completion and evaluation of the task, the new Teaching interaction model, the establishment of a new type of teacher-student relationship, the student's learning situation has been significantly improved, learning HRMS series of interest has been significantly improved, and enhance the students' sense of cooperation and all aspects of the ability.

\section{Acknowledgements}

Fund Project: Task-Driven Approach in Applied Undergraduate Human Resource Management Course; Project Number: NGJG-2015-01

\section{References}

[1] Gao Linhai.Application of task-driven in the teaching of human resources management in higher vocational colleges[J]. China Education Technology \& Equipment. 2016 (14)

[2] Dai Xiaoyun, Tian Xiao. Practice of Task-Driven Teaching Method in the Teaching of Recruitment and Recitation[J]. Popular Science and Technology. 2012 (02)

[3] ZHANG Dong-jie, WANG Qi-you, JIA Li-ping.Application of "Task-Driven Teaching Method" in the Teaching of Ideological and Political Theory in Colleges and Universities [J]. Journal of Agricultural University of Hebei (Agriculture and Forestry Education Edition)

[4] Li Dan. Task-driven teaching method in the use of high school ideological and political lessons [D]. Tianjin Normal University 2015

[5] Liu Yanji, Liu Yazhe.Study on the teaching reform of computer basic course in colleges and universities based on task driving method [J]. Management and Technology of Small and Medium sized Enterprises (Late Journal). 2014 (11) 